Hydroécol. Appl. (1992) Tome 4 Vol 2, pp. 149-158

\title{
Evolution météorologique et upwelling le long de la côte atlantique marocaine.
}

\section{Meteorological evolution and upwelling on the Atlantic Moroccan coast}

\author{
A. Agoumi ${ }^{(1)}$ et $A \cdot$ Orbi $^{(2)}$ \\ (1) E.H.T.P., BP 8108, Oasis Casablanca, (Maroc). \\ (2) I.S.P.M., 2, rue de Tiznit, Casablanca, (Maroc).
}

Résumé. - Cet article porte sur l'analyse de l'interaction entre des paramètres météorologiques et l'upwelling le long de la côte atlantique marocaine, particulièrement dans la région de Essaouira. La seconde partie est consacrée à l'analyse de l'évolution pluriannuelle des upwellings dans cette région en relation avec les vents mesurés in situ entre 1952 et 1988.

Abstract. - This work deals with the interaction between meteorological parameters and the upwelling along the Atlantic Maoroccan coasts, especially in the Essaouira area. An analysis of the in situ evolution of upwellings in that area is conducted taking into account the recorded winds during the period 1952-1988.

\section{INTRODUCTION}

La structure hydrodynamique du littoral atlantique Marocain se caractérise par la présence de plusieurs zones à upwelling (remontées d'eaux froides). On distingue globalement trois zones: la Zone Nord (région de larache), la zone Centrale (région d'Essaouira), la zone Sud (Tantan, Dakhla).

Les mécanismes de base qui conditionnent ces upwellings sont liés à la présence dans ces régions, en certaines périodes de l'année, de vents Nord-Est - Sud-Ouest (les Alizés): le cisaillement en surface dû à 
ces vents combiné à l'effet de Coriolis engendre un transport global des masses d'eaux côtières vers le large (transport d'Ekman). Ces eaux sont remplacées par des masses d'eaux froides et riches en éléments nutritifs, remontant du fond de l'océan.

Ce phénomène d'upwelling entraîne un enrichissement de la zone côtière en éléments nutritifs, ce qui favorise la production de l'écosystème littoral.
Différentes études ont été menées dans cette région et ont confirmé l'existence d'une relation étroite entre le développement et l'évolution des upwellings et la production de poissons. Ces études ont concerné en particulier les populations de sardines. On peut citer les travaux de Furnestin (1953, 1957, 1959, 1970), Grall et al. (1974), Minas et al. (1982), Belvese (1983, 1984), Orbi et al. (1991).

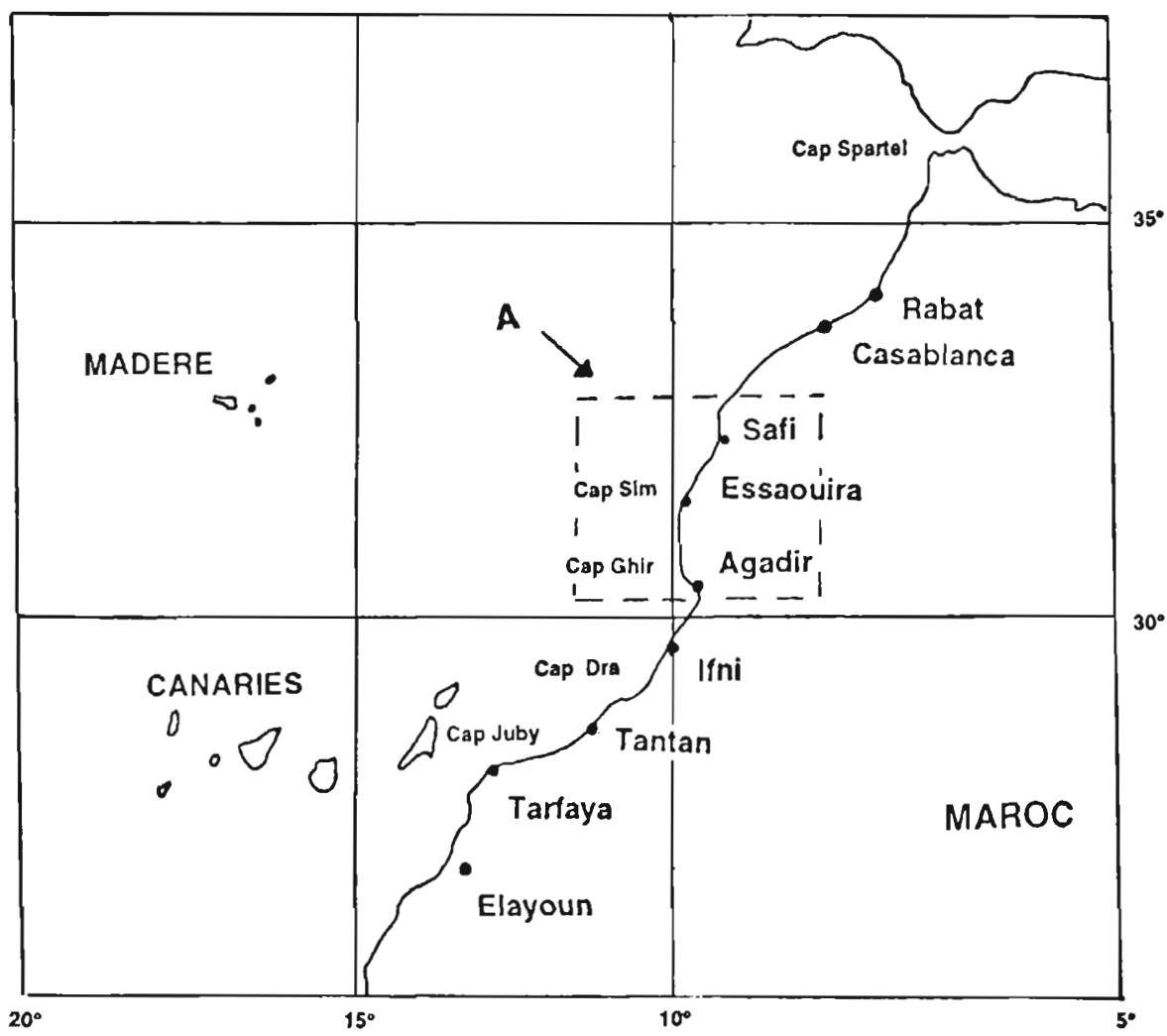

Fig. 1. - Situation géographique de la côte Nord-Ouest Africaine. 
Afin d'améliorer notre connaissance sur la dynamique d'apparition et de développement de ces upwellings en relation avec les paramètres climatiques de notre région, nous nous sommes fixés dans l'étude présentée ici deux objectifs:

1) étudier le phénomène d'upwelling à l'échelle saisonnière,

2) analyser son évolution pluriannuelle à partir des vents mesurés dans cette région.

L'analyse a porté sur une zone bien limitée: la région d'Essaouira (fig. 1). Les données utilisées sur les vents sont les intensités en $\mathrm{m} / \mathrm{s}$ et les directions en degré relevées à la station d'Essaouira chaque jour à 8 heures, 12 heures et 18 heures de 1952 à 1988.

\section{ÉVOLUTION SAISONNIÈRE}

A l'aide des mesures de vents relevées à la station d'Essaouira de 1952 à 1988 nous avons calculé l'indice d'upwelling moyen journalier. Ce premier calcul nous a permis de comparer l'évolution saisonnière différentielle de ce processus physique d'une année à l'autre:

L'indice d'upwelling moyen côtier d'une journée a été calculé en faisant le rapport du carré du module de la composante de la vitesse du vent parallèle à la côte au paramètre de Coriolis (Ben Jannet et al., 1989).

Le calcul des indices d'upwelling moyens journaliers nous a permis de tracer l'évolution de cet indice pour l'année moyenne de la période d'étude (1952, 1988) (Fig. 2).

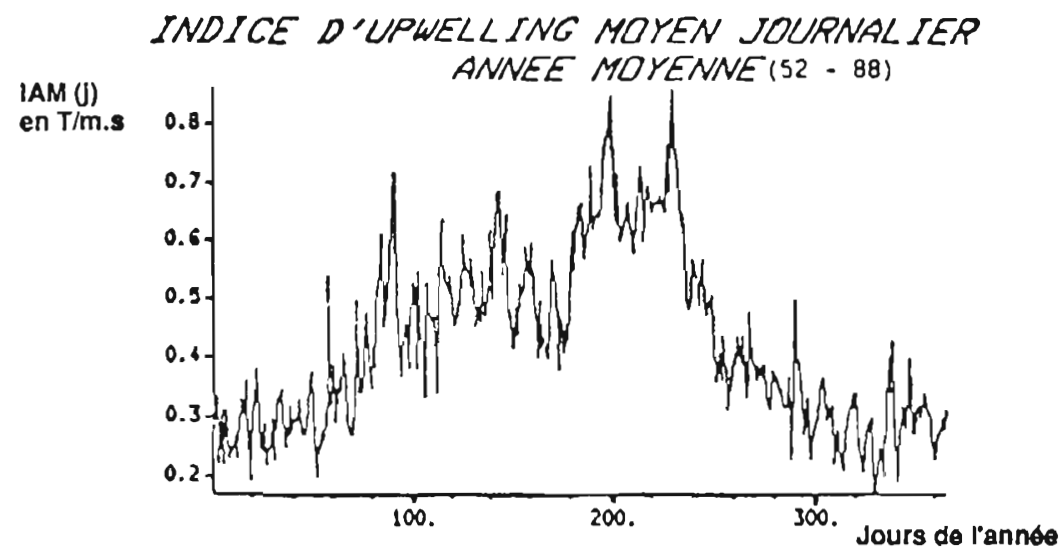

Fig. 2. - Indice d'upwelling journalier de l'année moyenne (T/s.m). 
L'indice moyen sur la période (IAM(j)) est calculé à partir des indices annuels journaliers $I_{A N}$ (j) sur la période 1952-1988.

$$
\operatorname{IAM}(j)=\sum_{A n=52}^{A n=88} I_{A N}(j) / 37
$$

$I_{A N}(j)$ : indice du jour $j$ de l'année AN. IAM(j): indice du jour j de l'année moyenne sur la période 1952-1988.

La tendance générale relevée sur cette année moyenne est que l'ordre de grandeur de l'indice d'upwelling est de 0,6 à 0,8 tonnes/m.s pendant l'été; l'installation de l'upwelling semble débuter vers le mois de mars pour atteindre son niveau le plus élevé aux mois de juillet-août; à partir de septembre l'upwelling commence à s'affaiblir.

L'évolution structurelle décrite cidessus reste cependant un indicateur moyen de l'évolution saisonnière du processus.

En pratique on s'aperçoit que certaines années s'écartent fortement de cette évolution moyenne.

En 1973, le niveau de cet indice est de l'ordre de 3 à $4 \mathrm{~T} / \mathrm{m}$.s pendant deux périodes de l'année (fig. 3) et voisin de 0 durant de nombreux épisodes.

En 1976, l'indice reste nul entre mai et début août et le niveau maximum annuel reste inférieur à $0,6 \mathrm{~T} / \mathrm{m} . \mathrm{s}$ (fig. 4).

En 1984, l'upwelling n'a pris naissance qu'en fin juin, en effet l'indice est resté inférieur à 1,5 T/m.s jusqu'à cette période (fig. 5 ).

\section{ÉVOLUTION PLURIANNUELLE}

Les données de vent relevées à la station d'Essaouira utilisées dans cette étude sont relatives à la période 1952-1988. Le calcul de l'indice d'upwelling à l'aide de ces séries de vitesse a permis d'associer à chacune de ces années un indice moyen annuel, défini comme la moyenne des valeurs journalières (IU).

Sur la figure 6 nous avons tracé l'évolution de cet indice d'upwelling moyen annuel pendant cette période. Sur les 37 années la moyenne annuelle est de l'ordre de 0,43 T/m.s. Le niveau de l'upwelling étant jusqu'en 1975 au voisinage sinon supérieur à cette moyenne (IU $=0,55 \mathrm{~T} / \mathrm{m} . \mathrm{s} 73$ ), entre 1976 et 1984 l'activité de cet upwelling a été très réduite (IU $=0,1 \mathrm{~T} / \mathrm{m} . \mathrm{s}$ en 76). Après cette phase l'upwelling a repris en $85-86$ pour connaître une nouvelle chute à partir de 1987: l'analyse de ce premier résultat nous amène à constater des fluctuations d'intensité très importantes ces dix dernières années par rapport à ce que la région connaissait les trentes années précédentes. On peut ainsi séparer au niveau climatique la période de temps étudiée 1952-1988 en deux phases: 19521975 avec un indice d'upwelling moyen de l'ordre de $0,49 \mathrm{~T} / \mathrm{m}$.s et 1976-1988 avec un indice d'upwelling moyen plus faible de l'ordre de $0,32 \mathrm{~T} / \mathrm{m} . \mathrm{s}$. Cette évolution se retrouve directement sur la figure 7 représentant les modules des vitesses moyennes annuelles de cette période. Dans la première phase ce module 
INDSCE D'UPWELLING MOYEN SOLPNAL IER

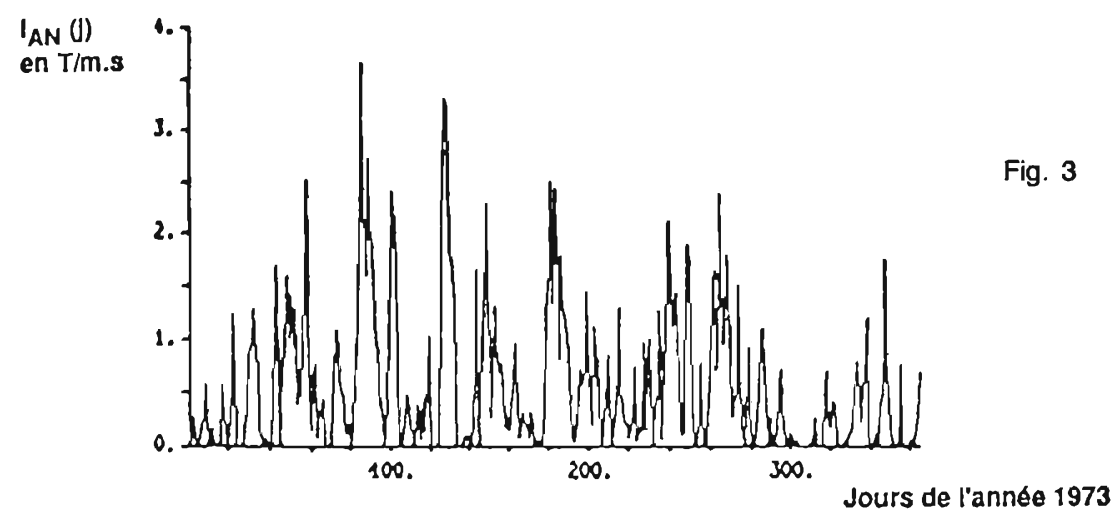

INDICE D'UPWELLING MOYEN JOURNAL IEP

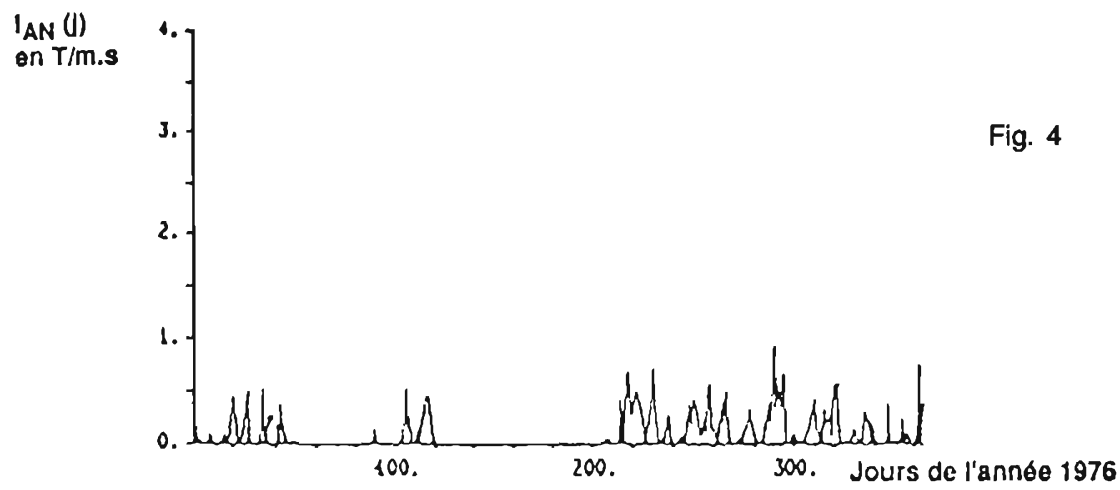

INDICE D'UPWELLING MOYEN SOURNAL IEP

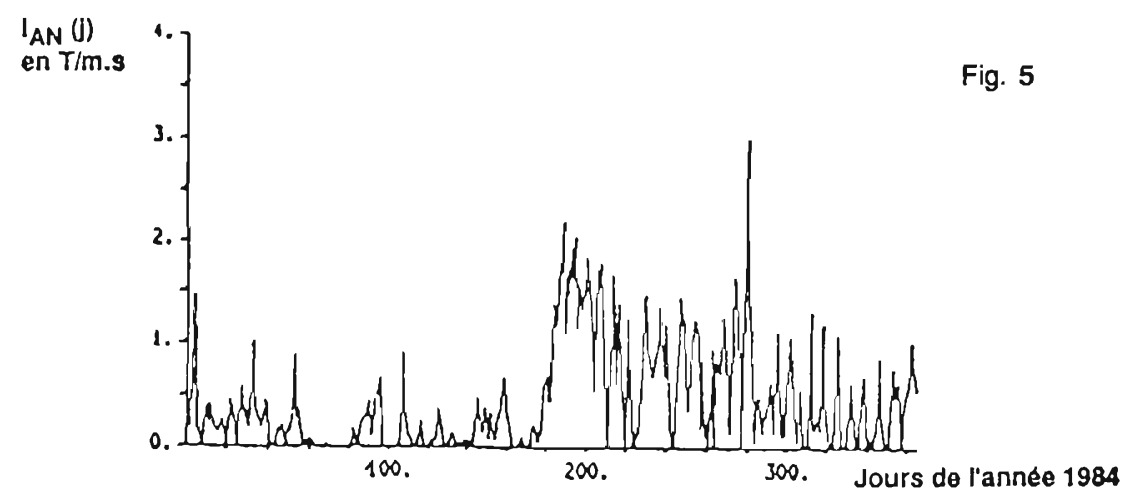

Fig. 3-4-5. - Indice d'upwelling journalier des années 73, 76 et 84 .

Fig. 3 (1973), Fig. 4 (1976), Fig. 5 (1984). 


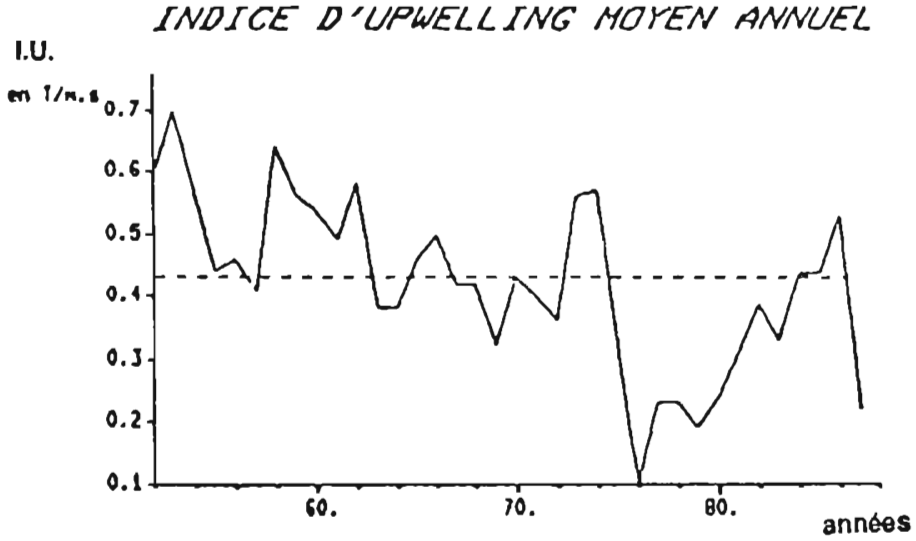

Fig. 6. - Indice d'upwelling moyen annuel de 1952 à 1988 (T/s.m).

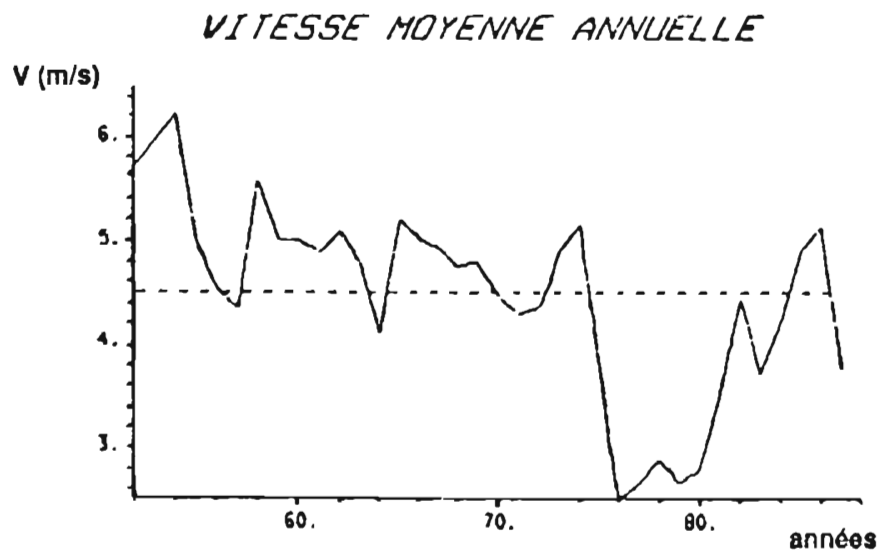

Fig. 7. - Vitesse moyenne annuelle de 1952 à $1988(\mathrm{~m} / \mathrm{s})$.

est pratiquement toujours supérieur à $4,5 \mathrm{~m} / \mathrm{s}$ alors qu'il est largement inférieur à cette valeur pendant toutes les années de la seconde phase (sauf en 87): l'intensité des vents est moins forte pour cette période (1976-1988) qu'elle ne l'était pour la période précédente (1952-1975).

Sur la figure 8 nous avons représenté la direction moyenne du vent des différentes années traitées. 


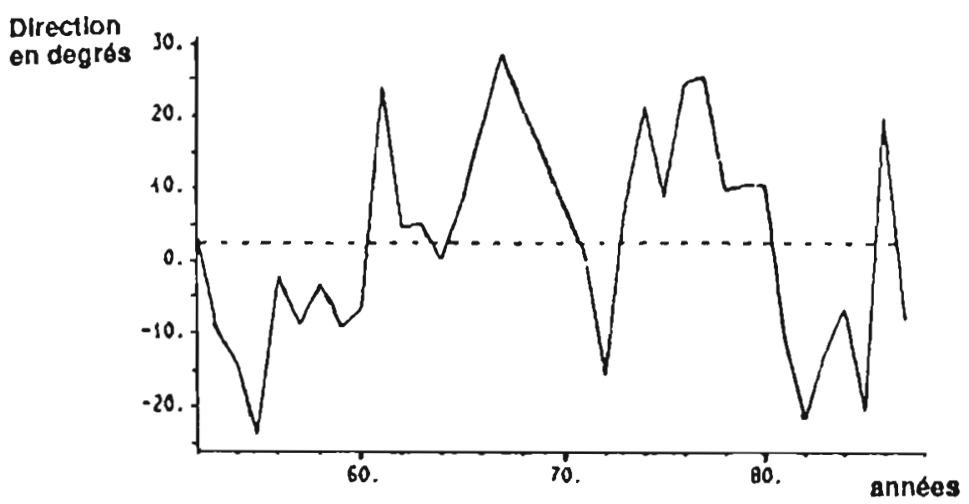

Fig. 8. - Direction moyenne annuelle du vent de 1952 à 1988 en degrés.

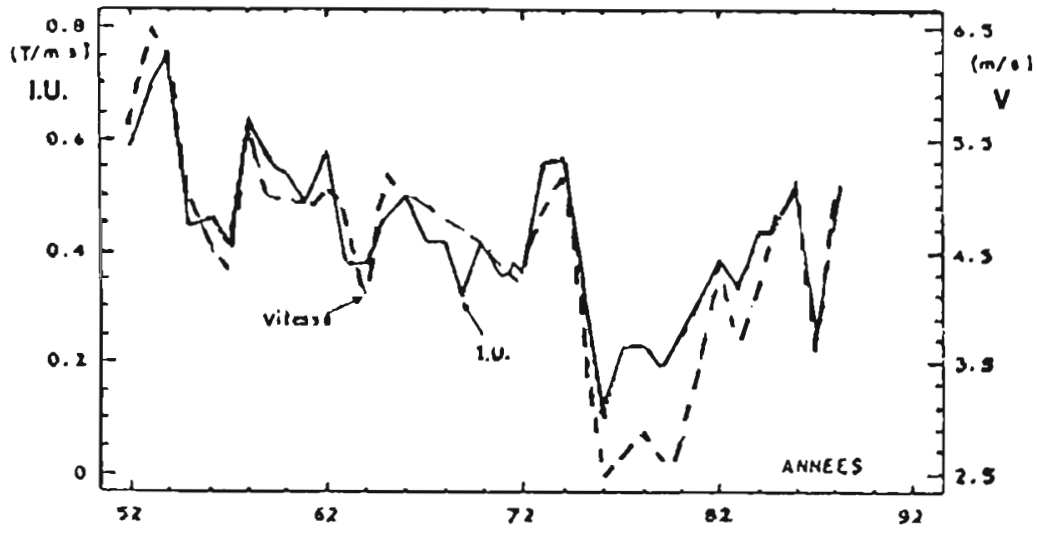

Fig. 9. - Vitesse et indice d'upwelling moyen annuel.

L'évolution de l'indice d'upwelling et du module du vecteur vitesse, qui sont représentés sur la figure 9 , semble similaire. Du fait que les vents largement dominants sont de secteur Nord, Nord-est et Nord-ouest (directions de 0 à 25 degrés), le vecteur vent n'est pas très différent de sa projection sur un axe parallèle à la côte, il en résulte que l'indice d'upwelling est fortement corrélé à la vitesse du vent: coefficient de corrélation de 0,93 (fig. 10).

Les fluctuations annuelles de l'indice d'upwelling ont été comparées à 


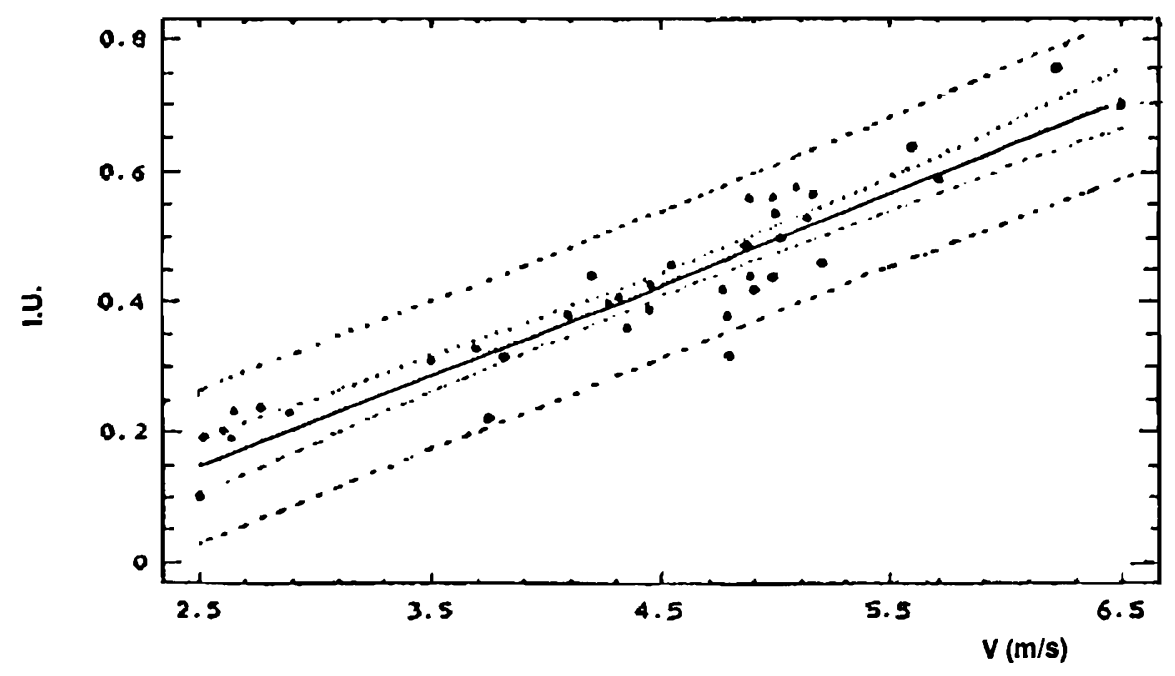

Fig. 10. - Régression linéaire entre l'indice d'upwelling et la vitesse moyenne annuelle.

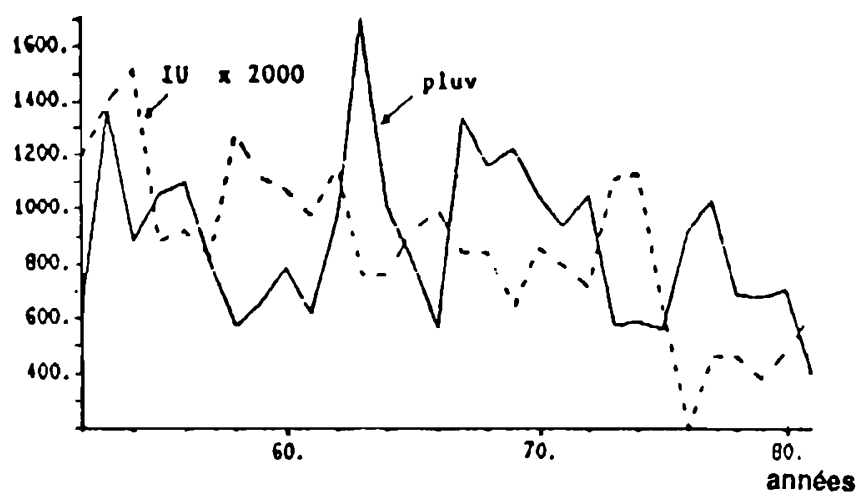

Fig. 11. - Indice d'upwelling moyen et pluviométrie annuelle à Essaouira + Safi.

un autre facteur climatique qui est la pluviométrie enregistrées à Essaoui$\mathrm{ra}+$ Safi (fig. 11). Une année de sécheresse à faible pluviométrie cor- respond systématiquement à un indice d'upwelling élevé: de 1957 à 1961 la pluviométrie est de l'ordre de $700 \mathrm{~mm}$ et l'indice d'upwelling est de 
0,55 T/m.s. En 1966 l'indice d'upwelling est de 0,5 et la pluviométrie de $600 \mathrm{~mm}$. En 1973-1976 la pluviométrie est de $600 \mathrm{~mm}$ et l'indice d'upwelling de $0,55 \mathrm{~T} / \mathrm{m} . \mathrm{s}$. Inversement les années pluvieuses sont des années où l'upwelling est faible notamment la période 1962-64, 66-71 et 74-80.

Cette analyse sommaire laisse supposer qu'il existe un lien étroit entre l'évolution climatique sur la côte atlantique marocaine et l'anticyclone des Açores: les années à fort upwelling sont des années pendant lesquelles cet anticyclone s'est maintenu longtemps au Nord engendrant des alizés sur une plus longue période de l'année et de ce fait empêchant le développement des vents du secteur Sud-Ouest chargés de pluies (années de sécheresse); inversement pour les années pluvieuses. L'anticyclone reste longtemps au niveau des Canaries. La période d'Alizés est réduite et donc le niveau d'upwelling est bas.

\section{CONCLUSION}

Cette étude nous a permis de confirmer le comportement moyen saisonnier de l'upwelling connu dans cette zone avec un début d'upwelling au printemps et un niveau maximum pendant l'été. Par contre, des écarts importants par rapport à cette moyenne ont été constatés pour des années particulières telle que 73-76-84.

L'étude globale de l'évolution interannuelle du vent en module et en direction pour la période 52-88 montre une baisse de ces vents à partir de 1975: les vents dominants dans cette zone sont essentiellement des vents du Nord engendrant l'upwelling côtier.

La période d'occurence de celui-ci est tributaire de la position de l'anticyclone des açores. Lorsque cet antjcyclone se maintient au niveau des îles Canaries, il engendre des vents du secteur Sud (chargés de pluies) et par la même occasion empêche le développement des vents du Nord qui sont le moteur de l'upwelling. La situation inverse est aussi retrouvée : les années de sécheresse, à fort upwelling, sont également de bonnes années pour la pêche.

L'importance de ce phénomène pour la dynamique des populations de poissons sur notre côte a été mise en évidence dans différents travaux de recherches, Orbi et al. (1991) ont mis en évidence celà en confrontant l'indice d'upwelling moyen annuel à Essaouira au CPUE (Captures par unité d'effort) des sardines pêchées dans la région entre 1968 et 1982. (fig. 12).

Celà devrait nous inciter à mener un effort de recherches important sur ce phénomène et son évolution sur nos côtes. II serait en particulier intéressant d'étendre cette étude à d'autres zones: zone nord, zone sud (Dakhla et Tan-Tan).

\section{REMERCIEMENTS}

Ce travail a pu être réalisé grâce à la collaboration de la Direction de la Météorologie Nationale. Nous tenons ici à remercier les ingénieurs de cette direction pour leur participation. 


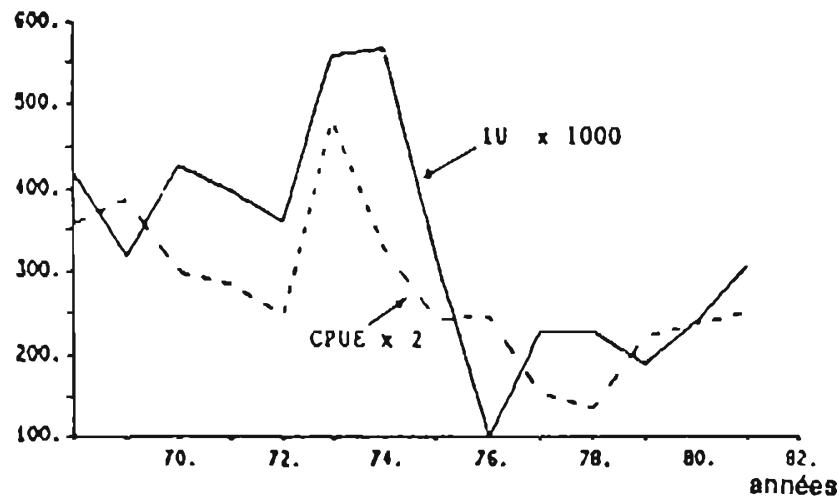

Fig. 12. - CPUE (en tonnes) et indice d'upwelling moyen annuel de 1968 à 1981. (ORBI et al (1991)) IUP (en T/M.s).

\section{BIBLIOGRAPHIE}

Belvèze $\mathrm{H}$. and Erzini K., 1983. Influence of hydroclimatic factors in the availability of sardine (Sardina pilchardus walb.) in the moroccan fisheries in the Atlantic. Expert Consultation to examine changes in abondance and species composition of Neretic stocks, paper $\mathrm{n}^{\circ}$ 25. San jose, Costa Rica. April 1983.

Belveze H., 1984. Biologie et dynamique des populations de sardines, Sardina pilchardus walb peuplant les côtes Atlantiques Marocaines et proposition pour un aménagement des pêcheries. Thèse Université de Bretagne Occidentale, Brest, France: $532 \mathrm{p}$.

Ben Jannet G. et Tazi S., 1989. Pêcheclimat en zone d'upwelling (côte Atlantique Marocaine). Projet de fin d'étude. Ecole Hassania des travaux publics, 140 p. Mai 89.

Furnestin J., 1953. Ultra-sons et pêche de la sardine au Maroc. Bull. Ins. Pêches. Maroc, $n^{\circ} 15$.

Furnestin J., 1957. Le milieu. Revue. Trav. Inst. Pêch. Marit. 21: (1 et 2): 19-38.

Furestin J., 1959. Hydrologie du Maroc Atlantique. Revue. Trav. Ins. Pêch. Marit. 23: (1) 5-77.
Furestin J., Furestin M.L., 1970. La sardine marocaine et sa pêche. Migration trophique et génétique en relation avec l'hydrologie et le plancton. Rapp. P.-V. Reun. Cons. Int. Explor. Mer. 159: 165175.

Grall J.R. et al., 1974. Caractéristiques trophiques et production planctonique dans la région Sud de l'Atlantique Marocain. CINECA-Charcot I et II. Téthys 6, (1-12): 11-28.

Grall J.R., Le Corre P. et Tréguer P., 1982. Short-term variability of primary production in coastal upwelling of Morocco. Rapp. P.V. Reun. Cons. Inst. Explor. Mer. 180: 221-227.

Minas H.J. et al., 1982. Nutrients and primary production in the upwelling region of north west Africa. Rapp. P.-V. Reun. Cons. Int. Explor. Mer. 180: 148-183.

Orbi A., Agoumi A., Ben Jannet G., Tazi S., 1991. Approche modélisatrice des fluctuations d'abondance des sardines sur la côte Nord-Atlantique Marocaine, dans les pêcheries Ouest-Africaines, variabilité, instabilité et changement. Cury, Roy 1991. 